\title{
BMJ Open Does app-based unguided self- management improve mental health literacy, patient empowerment and access to care for people with mental health impairments? Study protocol for a randomised controlled trial
}

\author{
André Kerber (D) , ${ }^{1}$ Ina Beintner, ${ }^{2}$ Sebastian Burchert, ${ }^{1}$ Christine Knaevelsrud ${ }^{1}$
}

To cite: Kerber A, Beintner I, Burchert S, et al. Does app-based unguided selfmanagement improve mental health literacy, patient empowerment and access to care for people with mental health impairments? Study protocol for a randomised controlled trial. BMJ Open 2021;11:e049688. doi:10.1136/ bmjopen-2021-049688

- Prepublication history and additional online supplemental material for this paper are available online. To view these files, please visit the journal online. To view these files, please visit the journal online (http://dx.doi.org/10.1136/ bmjopen-2021-049688).

Received 29 January 2021 Accepted 24 June 2021

Check for updates

(c) Author(s) (or their employer(s)) 2021. Re-use permitted under CC BY-NC. No commercial re-use. See rights and permissions. Published by BMJ.

${ }^{1}$ Division of Clinical Psychological Intervention, Department of Education and Psychology, Freie Universität Berlin, Berlin, Germany

${ }^{2}$ MindDoc Health GmbH, Munich, Germany

Correspondence to

André Kerber;

andre.kerber@fu-berlin.de

\section{ABSTRACT}

Introduction Mental disorders pose a huge burden to both individuals and health systems. Symptoms and syndromes often remain undetected and untreated, resulting in comorbidity and chronification. Besides limited resources in healthcare systems, the treatment-gap is- to a large extent-caused by within-person barriers impeding early treatment seeking. These barriers include a lack of trust in professionals, fear of stigmatisation, or the desire to cope with problems without professional help. While unguided self-management interventions are not designed to replace psychotherapy, they may support early symptom assessment and recognition by reducing withinperson barriers. Digital self-management solutions may also reduce inequalities in access to care due to external factors such as regional unavailability of services. Methods and analysis Approximately 1100 patients suffering from mild to moderate depressive, anxiety, sleep, eating or somatisation-related mental disorders will be randomised to receive either a low-threshold unguided digital self-management tool in the form of a transdiagnostic mental health app or care as usual. The primary outcomes will be mental health literacy, patient empowerment and access to care while secondary outcomes will be symptom distress and quality of life. Additional moderator and predictor variables are negative life events, personality functioning, client satisfaction, mental healthcare service use and application of selfmanagement strategies. Data will be collected at baseline as well as 8 weeks and 6 months after randomisation. Data will be analysed using multiple imputation and analysis of covariance employing the intention-to-treat principle, while sensitivity analyses will be based on different multiple imputation parameters and a perprotocol analysis.

Ethics and dissemination Approval was obtained from the Ethics Committee of the Faculty of Educational Science and Psychology at the Freie Universität Berlin. The results will be submitted to peer-reviewed specialised journals and presented at national and international conferences. Trial registeration The trial has been registered in the DRKS trial register (DRKS00022531);Pre-results.
Strengths and limitations of this study

- This study is the first to systematically investigate treatment seeking attitudes and behaviours in a large population of users of an app-based mental health intervention.

- The assessment of both predictors of treatment seeking and actual treatment seeking behaviour enables the investigation of possible indicators for decision making in stepped care.

- The inclusion of a control group, possible moderator variables and follow-up measurements will allow analyses to assess potential mechanisms of improvement.

- The study may be limited by a relatively high rate of attrition that is to be expected in unguided self-help solutions.

\section{INTRODUCTION}

The number of people suffering from depression worldwide is estimated at over 300 million and more than 250 million suffer from an anxiety disorder. ${ }^{1}$ In Germany, around 1 in 10 people is affected by depression, while around one in five meets diagnostic criteria for an anxiety disorder. ${ }^{2}$ One in 20 individuals suffers from chronic pain ${ }^{2}$ or insomnia. ${ }^{3}$ Depressive disorders are a major contributor to health loss, accounting for $7.5 \%$ of years lived with disability while anxiety disorders rank in the top ten among all known diseases ${ }^{14}$ accounting for $4.5 \%$ of years lived with disability. ${ }^{14}$. Furthermore, mental disorders are associated with high direct and indirect costs. In Germany, the direct costs in 2012 were estimated at 33 billion Euro $^{5}$ while estimates of indirect costs are almost equally high. ${ }^{6}$ 
Further, comorbidity among mental disorders is high. For example, individuals with a mood disorder, e.g. depression, have a lifetime prevalence of $81 \%$ for anxiety disorders, with social phobia, obsessive compulsive disorder, generalised anxiety disorder or post-traumatic stress disorder being the most frequent comorbid disorders. ${ }^{7}$ A review of 177 clinical studies with a total of 533377 study participants revealed that only $14 \%$ of the cases could be clearly allocated to one specific mental disorder category such as depression, anxiety or personality disorders. ${ }^{8}$ When affective and anxiety disorders are conceptualised under the overarching spectrum of internalising disorders, predictive validity with respect to suicidal tendencies or future mental illness is improved significantly. ${ }^{9}$ A similar approach is used in contemporary dimensional, hierarchical and datadriven phenotypic definitions of psychopathology ${ }^{10}$ which is also supported by recent findings concerning shared genetic covariance and polygenic risk scores. ${ }^{11}$

Recent meta-analyses confirm the efficacy of appsupported smartphone interventions both for the reduction of common mental disorder symptoms as well as for improving quality of life. ${ }^{12}$ These interventions are typically based on principles of cognitive-behavioural therapy and are designed to teach the users skills to manage their symptoms as well as disorder-related cognitions and behaviours. ${ }^{13}$

Typically, internet-based interventions consist of several sessions or modules and address one type of disorder. ${ }^{13}$ Interventions targeting the same disorder tend to be very similar regarding their components and content, while they may differ in the way the content is presented (eg, text vs video based, length and reading level of text, inclusion of case vignettes and examples). Sessions or modules can be consecutive, that is, users engage with the content in a preset order and/or at preset intervals, or users can determine the order of the content they engage with and self-pace through the intervention.

On the other hand, authors of an extensive analysis of the WHO World Mental Health Surveys conclude that 'common causal pathways account for most of the comorbidity'. ${ }^{14}$ This may explain why many pharmaceutical and psychosocial treatments show transdiagnostic effects on a range of mental disorders. ${ }^{15} 16$ Contemporary mental health interventions and treatments such as the Unified Protocol ${ }^{17}$ or the Common Elements Treatment Approach ${ }^{18}$ therefore increasingly replace 'singledisorder protocols' with treatment elements that address the common underlying mechanisms of multiple disorders and have been proved to be transdiagnostically effective.

Based on these as well as the meta-analytical findings presented above we therefore expect a transdiagnostic unguided internet based self-management application for mental health to have an impact on a range of mental disorder symptoms beyond anxiety and depression as well as on quality of life.

Most common mental disorders can be successfully treated if they are detected early and if appropriate treatment is provided in a timely manner. To this end, evidence-based therapeutic approaches are available but only reach about $28 \%$ (in high-income countries) of those with depression and $20 \%$ of those with an anxiety disorder. ${ }^{19}$ Access to specialised care is often impeded by limited availability while treatment delays are associated with symptom deterioration and less favourable long-term outcomes, according to a study using longitudinal data from over half a million treatment-seeking individuals. ${ }^{20}$

More importantly, within-person attitudinal barriers seem to constitute an even stronger obstacle for treatment seeking than structural barriers. Both a major national population study ${ }^{21}$ and the WHO World Mental Health surveys $^{22}$ concluded that by far the largest treatment barriers are wanting to handle the problem on one's own and low perceived need for care. Although not considered to be stigma-related barriers, these factors may be influenced by stigma. ${ }^{23} \mathrm{~A}$ systematic review of barriers and facilitators to mental health help-seeking showed the key barriers to be stigma, confidentiality issues, lack of accessibility, self-reliance, low knowledge about mental health services and fear/stress about the act of help-seeking or the source of help itself. ${ }^{24}$ Consequently, stigma can be considered a part of a larger network of beliefs and other constraints deterring help-seeking behaviour. ${ }^{25}$ All of these factors reduce the chances of early detection, add to the issue of under-diagnosis, and increase the risk of long-term symptom deterioration and chronification. ${ }^{26}$

Low threshold digital mental health interventions have been found to increase patients' self-management skills, improve the communication to healthcare providers, have the potential to reach non-treatment-seeking patient groups and could foster destigmatisation. ${ }^{27} 28$ The impact of psychoeducative or web-based self-help interventions on within-person attitudinal barriers has also been shown quantitatively. ${ }^{29}{ }^{30}$ A number of studies evaluating digital mental health interventions for depression and anxiety have shown a decrease in (self-) stigmatisation. ${ }^{31-33}$ Effects on help seeking attitudes and actual help seeking have been detected in several randomised controlled trials (RCTs). ${ }^{33-36}$ Those effects mostly are associated with effects on health literacy, which also has been shown in previous studies. ${ }^{31} 33$ Apart from effects that may facilitate help seeking behaviour, digital mental health interventions have been found to increase the application of self-management skills ${ }^{34}$ and were found to have positive effects on subjective health-ratings. ${ }^{35}$

A qualitative study investigating the potential utility of mental health app components on reducing helpseeking barriers, ${ }^{37}$ suggested that self-assessment with individualised feedback, informative videos by mental health professionals, testimonials from mental health service users, and a platform for an online connection with a professional have a potential to reduce withinperson barriers to help seeking. Most of these components, especially psychoeducative elements, are central parts of unguided app-based self-management for mental health. 
Based on these findings, one can expect that the use of a transdiagnostic unguided internet based selfmanagement for mental health may lead to significant improvements both in health literacy and variables that reflect patient empowerment, such as help-seeking, reduced stigma and self-management behaviours.

While self-management interventions cannot replace psychotherapy and are not designed to do so, they can support assessment and recognition, reduce withinperson barriers as well as treatment gaps and inequalities and facilitate self-management of symptoms and problems. The MindDoc App constitutes such a transdiagnostic, low-threshold monitoring and selfmanagement application aimed at people with mild to moderate expressions of mental disorders from the internalising spectrum. It provides automated tailored feedback and suggests psychological exercises based on reported symptoms and problems, but also allows users to access psychological exercises at their own discretion.

Taken together, we therefore hypothesise that the use of the MindDoc App in addition to care as usual (CAU) is associated with an increase in mental health literacy, patient empowerment and facilitation of access to care, compared with CAU alone. Furthermore, this study aims to explore whether the use of the MindDoc App in addition to CAU leads to a greater reduction of psychopathological symptom load of mental disorders of the internalising spectrum and a stronger improvement in quality of life.

\section{METHODS AND ANALYSIS}

\section{Study design}

To examine the effects of MindDoc App usage on health literacy, patient empowerment, access to care, symptom distress and quality of life, participants will be randomly assigned to either the intervention group or the CAU group following the baseline assessment. The intervention group will receive immediate access to all features and courses included in the MindDoc App. The CAU group will receive access to the MindDoc App after 6 months (ie, after the follow-up assessment).

In total, there will be three measurement points (aside from the continuous assessment of app usage behaviour): Baseline (initial diagnostics), post (after 8 weeks), and follow-up (after 6 months). An overview on the measurements and measurement points is provided in table 1 , the study process is visualised in figure 1 .

\section{Patient and public involvement}

Patients or the public were not involved in the design, or conduct, or reporting, or dissemination plans of our research, although user/patient feedback was an important source for the development and improvement of the mental health app investigated in this trial.

\section{Intervention}

Users in the intervention group will get immediate access to the MindDoc App, which provides a monitoring tool that allows users to track symptoms of common mental

Table 1 Overview of assessments and study visits

\begin{tabular}{|c|c|c|c|}
\hline & Baseline & $\begin{array}{l}\text { Postintervention } \\
\text { ( } 8 \text { weeks after } \\
\text { baseline) }\end{array}$ & $\begin{array}{l}\text { Follow-up } \\
\text { ( } 6 \text { months after } \\
\text { baseline) }\end{array}$ \\
\hline Questions assessing key sociodemographic variables & \multicolumn{3}{|l|}{$x$} \\
\hline Questions assessing whether inclusion and exclusion criteria are fulfilled & \multicolumn{3}{|l|}{$\mathrm{x}$} \\
\hline PHQ-9 & \multicolumn{3}{|l|}{$x$} \\
\hline GAD-7 and MINI-SPIN & \multicolumn{3}{|l|}{$\mathrm{x}$} \\
\hline PHQ-15 & \multicolumn{3}{|l|}{$\mathrm{x}$} \\
\hline RIS & \multicolumn{3}{|l|}{$\mathrm{x}$} \\
\hline PID5BF+and OPD-SQS & \multicolumn{3}{|l|}{$x$} \\
\hline AQoL 8-D & \multicolumn{3}{|l|}{$\mathrm{x}$} \\
\hline MHLq & \multicolumn{3}{|l|}{$x$} \\
\hline $\begin{array}{l}\text { Assessment of Mental Health-Related Patient Sovereignty and Self } \\
\text { Management Strategies }\end{array}$ & $\mathrm{x}$ & \multicolumn{2}{|l|}{$\mathrm{x}$} \\
\hline IASMHS & $\mathrm{x}$ & $\mathrm{x}$ & $x$ \\
\hline Healthcare Service Use Questionnaire & \multicolumn{2}{|l|}{$x$} & $x$ \\
\hline \multicolumn{2}{|l|}{ CSQ-I } & $x$ & $x$ \\
\hline \multicolumn{2}{|l|}{ LES and (serious) adverse events } & & $x$ \\
\hline \multicolumn{4}{|c|}{$\begin{array}{l}\text { AQoL, Assessment of Quality of Life; CSQ-I, Client Satisfaction Questionnaire adapted to internet based interventions; GAD, Generalized } \\
\text { Anxiety Disorder Scale; IASMHS, Inventory of Attitudes Toward Seeking Mental Health Services; LES, Life Events Scale; MHLq, Mental } \\
\text { Health Literacy Questionnaire; MINI-SPIN, Mini Social Phobia Inventory; OPD-SQS, Operationalized Psychodynamic Diagnosis - Structure } \\
\text { Questionnaire Short; PHQ, Patient Health Questionnaire; PID5BF+, Personality Inventory for DSM-5 Brief Form Plus; RIS, Regensburg } \\
\text { Insomnia Scale. }\end{array}$} \\
\hline
\end{tabular}




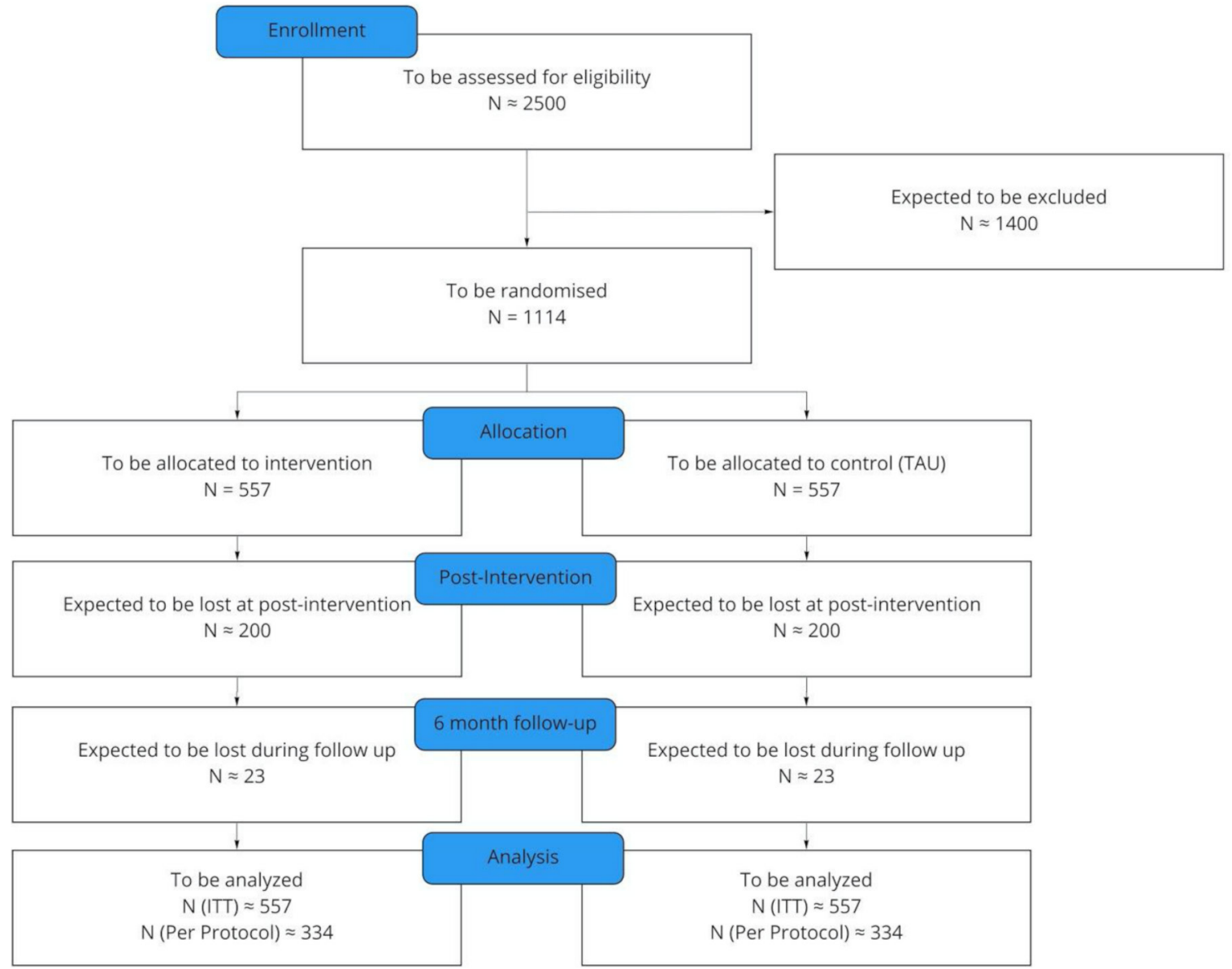

Figure 1 Study process and participant timeline. ITT, intention to treat.

health problems over long time periods. The application has four core components which are interconnected to deliver the described benefit for the user: (1) continuous monitoring of symptoms of common mental disorders, related problems and personal resources (Journal), (2) biweekly automated feedback on general symptom load and level of functioning (Results), (3) continuous automated feedback on symptoms, symptom clusters and relevant problem areas, along with recommendations for exercises and courses (Insights) and (4) Structured selfmanagement courses and exercises that address problems that commonly contribute to mental health disorders (SelfManagement). Questions are asked within three blocks a day (morning, noon, evening), with each block consisting of three or more questions. The underlying algorithm adjusts the number and area of questions to the answers of the user as well as to the completion rate of previous questions blocks. Every question block is followed by a general mood-tracking (very bad, bad, moderate, good, very good) as well as the opportunity to track emotions and situations via text entry and predefined or customised tags (positive, negative, neutral). This information is then processed to continuously provide individualised automated feedback (Insights) to the user that reflect symptoms as well as potential triggers and problem areas and personal resources and suggest suitable disorderspecific and transdiagnostic self-management courses and exercises to address symptoms and problems. As soon as the user has answered the required minimum of questions, the application provides an individualised medical orientation regarding the need for assessment by a specialist. Research on a previous version of the MindDoc App yielded good diagnostic accuracy compared with a gold-standard measure for depression. ${ }^{38}$

In case a user indicates suicidal ideation within the monitoring feature of the application, a crisis chat bot is immediately activated that directs the user to a national crisis helpline (in Germany: Telefonseelsorge) that can be called directly from the app.

Users in the CAU group will get access to the MindDoc App after completing the final assessment. The MindDoc App was developed and is provided by MindDoc Health $\mathrm{GmbH}$, a subsidiary of Schoen Clinic, which is a hospital group in Germany with one specialisation in mental health. First published in 2016 under the name Moodpath, it was mainly directed at individuals with depressive disorders. Since 2019, the scope has been expanded to include other common mental disorders, resulting in an extension of both the monitoring system and the content library. Since October 2020, the App is available under the name MindDoc. All content was developed under the supervision of IB, who is a licensed clinical psychologist with a research background in e-mental-health. The theoretical foundation of the monitoring system is the 
Hierarchical Taxonomy of Psychopathology, ${ }^{39}$ courses and exercises are based on national and international treatment guidelines. A detailed description of the MindDoc App can be found in online supplemental material.

\section{Measures}

Psychopathology

The PHQ-9 (Patient Health Questionnaire, 9-item depression subscale) is the depression module of the selfadministered version of the PRIME-MD (Primary Care Evaluation of Mental Disorders) diagnostic instrument for common mental disorders. It scores each of the 9 DSM-5 (Diagnostic and Statistic Manual of Mental Disorders, 5th edition) diagnostic criteria as 0 (not at all) to 3 (nearly every day). The PHQ-9 is a reliable (Cronbach's alpha $=0.89)$ and valid measure of depression severity. ${ }^{40}$

The GAD-7 (Generalized Anxiety Disorder Scale, 7-items) is a one-dimensional instrument designed to detect symptoms of generalised anxiety disorder as it is defined in the DSM-5. The item scores range from 0 (not at all) to 3 (nearly every day). The GAD-7 is a valid and efficient tool for screening for anxiety disorders and assessing its severity in clinical practice and research. ${ }^{41}$

The PHQ-15 is the module for the severity of somatic symptoms of the self-administered version of the PRIME-MD diagnostic instrument for common mental disorders. It comprises 15 somatic symptoms from the PHQ each symptom scored from 0 ('not bothered at all') to 2 ('bothered a lot'). The PHQ-15 is a reliable (Cronbach's alpha $=0.80$ ) and valid screening tool for somatisation. ${ }^{42}$

The Regensburg Insomnia Scale $\left(\mathrm{RIS}^{43}\right)$ is a self-rating scale to assess cognitive, emotional and behavioural aspects of psychophysiological insomnia (PI) with ten items. It has good internal consistency with Cronbach's alpha $=0.89$ and distinguishes well between controls and patients with PI.

The PID5BF+(Personality Inventory for DSM-5, Brief Form Plus) is a short form of the personality inventory for DSM-5 (PID-5) with 34 items, which is also compatible with the dimensional assessment of maladaptive personality expressions in the ICD-11. The OPD-SQS (Operationalized Psychodynamic Diagnosis - Structure Questiona Short) is a short 12-item assessment for the severity of personality dysfunction. Dimensional assessment of severity and style of personality dysfunction according to DSM-5 and ICD-11 (International Classicifation of Diseases, 11th version) are important predictors of treatment course, adherence, response, and general psychopathology. ${ }^{44}$ Both the OPD-SQS (Cronbach's alpha $=0.89$ ) and the PID5BF+ (average McDonald's Omega $=0.81$ ) are validated and reliable measures. ${ }^{45-47}$

\section{Quality of life}

The Assessment of Quality of Life-8D is a multiattribute 35-item self-rating scale which was constructed for the evaluation of health services that have an impact on the psychosocial aspects of the quality of life. It comprises the assessment of six psychosocial functioning domains as well as the physical autonomy. It demonstrated good reliability (Cronbach's alpha $=0.96$ ) and convergent and predictive validity. ${ }^{48}$

\section{Mental health literacy, patient empowerment and help seeking}

The Mental Health Literacy Questionnaire (MHLq) is a 29-item scale which assesses mental health literacy on four dimensions (knowledge of mental health problems, erroneous beliefs/stereotypes, help-seeking and first aid skills, self-help strategies). Scores showed significant differences between individuals with more or less experience with mental health as well as good internal consistency (Cronbachs Alpha $=0.84$ ) for the total score. ${ }^{49}$

Assessment of mental health-related patient sovereignty and self management strategies (AMHPSSS): Based on a systematic review on self-management strategies for depression, ${ }^{50}$ a Delphi consensus study on self-help strategies for depression ${ }^{51}$ as well as two studies on useful self management strategies for $\operatorname{mood}^{52}$ and anxiety ${ }^{53}$ disorders from the patient perspective, we identified 18 useful self-management strategies that were replicated at least once from expert and patient perspectives. We then formulated these strategies in questionnaire format asking for the frequency of application of the respective strategy in the last 8 weeks on a 5-point Likert scale, for example, 'in the last 8 weeks, you engaged in activities that gave you a feeling of achievement'. We then further extracted strategies and behaviours that are indicators of patient sovereignty according to a conceptual framework for patient choice and empowerment in northern European health systems. ${ }^{54}$ This resulted in 10 items in statement format asking how much participants agree or disagree on a 5-point Likert scale, for example, 'I know well about the treatment options for my disease'.

The Inventory of Attitudes Toward Seeking Mental Health Services (IASMHS) is a 24-item scale assessing three internally consistent within-person barriers to seeking mental health services: Psychological openness, help-seeking propensity and indifference to stigma. Internal consistency (Cronbach's alpha $=0.87$ ) and validity of the assessment could be confirmed in separate samples. ${ }^{55}$

Mental Health Service Use Questionnaire: Based on expert consensus between three licensed psychotherapists and one psychiatrist, a list comprising digital mental health interventions, alternative/complementary methods as well as preventive, psychosocial and therapeutic/curative/professional services with a total of 21 items was generated. Participants will be asked which services/interventions they used how often in the last 6 months.

\section{Satisfaction with the APP and usage behaviour}

The Client Satisfaction Questionnaire adapted to Internet-based interventions is a measure to assess satisfaction with web-based health interventions with a onefactorial structure and eight items. It demonstrated good 
model fit, reliability $($ McDonalds Omega $=0.93)$ and correlated significantly with change in depressive symptoms and perceived stress. ${ }^{56}$

Data assessed within the MindDoc App: Within the MindDoc App, usage data and responses within the monitoring system are stored and may be used for secondary analyses. Data from the two sources (MindDoc App, Study Survey) will be consolidated via a personalised download link which users in the intervention group receive after randomisation.

\section{Other measures}

The Life Events Scale is a list of 42 major life events such as divorce, change in residence or a child leaving home adapted from Holmes and Rahe ${ }^{57}$ and Hobson et a $\tilde{l}^{58}$. Major life events are important predictors for occurence and relapse of mental health problems. Participants are asked to indicate whether a major life event occurred within the time of the study or in the 6 months before and how much they were affected by the life event.

\section{Adverse events:}

If the crisis chat bot within the app is triggered, or if a participant scores $>1$ on the PHQ-9 suicidality item, this will be recorded as an adverse event (AE). Other AEs and serious AEs (SAEs) will be collected via self-report during the post-intervention and follow-up assessments.

\section{Sample size}

The primary endpoint is the change in MHLq, selfmanagement skills and patient sovereignty (AMHPSSS) and within-person barriers to help seeking (IASMHS) after 8 weeks and a change in actual help-seeking behaviour after 6 months compared with baseline. Based on a metaanalysis on the efficacy of app-supported smartphone interventions for mental problems ${ }^{12}$ as well as a review of the very few previous RCTs investigating the effects of digital mental health interventions on help seeking attitudes, general practitioner visits and mental health literacy. ${ }^{33-36}$ We expected a small to moderate effect on the primary outcomes of $d=0.3$ (Cohen's $d$ ). Based on a metaanalysis on attrition rate in smartphonedelivered interventions for mental health problems, ${ }^{59}$ we conservatively estimated a dropout rate of $40 \%$.

Using a multivariate (intersection-union) z-test to detect the expected difference between the intervention and the CAU control group with a probability of $90 \%$ at a significance level of $\alpha=2,5 \%$, estimated intercorrelation of .5 between these multiple primary endpoints and a dropout rate of approx $40 \%$, a total sample of $\mathrm{N}=1113$ subjects is necessary.

\section{Recruitment}

Participants will be recruited via press releases and social media as well as health insurance member magazines and websites. Recruitment will take place over a period of approximately 1 year (2021). Participation in the study can be anonymous, but the participants must provide an (anonymous) email address at which they can be contacted. Anonymous participation is associated with a lower threshold for engaging in mental health research.

\section{Eligibility criteria}

\section{Inclusion criteria}

Eligible for the trial are participants with symptoms of internalising disorders indicated by scoring above the cut-off on one or more of the following scales: PHQ-9 score $>4$ or GAD-7 score $>4$ or MINI-SPIN score $>6$ or PHQ-15 score $>4$ or binge eating or compensatory behaviours $>$ once/ week or body mass index (BMI) $<18.5 \mathrm{~kg} / \mathrm{m} 2$ or critical weight loss and weight and shape concern or RIS score $>12$.

In addition, eligible participants need to have full legal capacity (self-disclosure), access to a smartphone (iOS or Android) and the internet (self-disclosure) and residence in Germany (self-disclosure).

\section{Exclusion criteria}

Not eligible for the trial are participants with too severe symptoms of internalising disorders indicated by acute suicidality: Score $>1$ on PHQ-9 item 9 or answer 'yes' to acute suicidality screening question or PHQ-9 $>19$ (severe depression $^{40}$ ) OR GAD-7 $>15$ (severe anxiety disorder ${ }^{60}$ ) OR PHQ-15 score $>14$ (severe symptoms according to ${ }^{42}$ ) or BMI $<15 \mathrm{~kg} / \mathrm{m} 2$.

In addition, participants currently in inpatient (selfdisclosure) or ongoing psychotherapeutic treatment (self-disclosure) or reported history of bipolar disorder, psychotic disorder, substance use disorder (selfdisclosure) or age $<18$ are excluded.

Participants meeting these exclusion criteria are given detailed information on treatment options.

\section{Procedures}

All assessments described in this study protocol will be carried out via online surveys in a separate web-based platform outside of the mental health app investigated in this trial (Unipark/EFS Survey). Before the baseline assessment, participants will receive written information about the study, the intervention, the randomisation, the assessments and the data processing. Once they have given their consent, they are given access to the baseline assessment, which also includes questions that assess their eligibility to participate in the study according to the predefined inclusion and exclusion criteria. If a participant is eligible and the baseline assessment is complete, the participant will be randomly assigned to either the intervention or the control group in a 1:1 ratio by an algorithm provided by the assessment platform (Unipark/EFS Survey). Participants in the intervention group then receive access to the MindDoc App and will be recommended to use it for 8 weeks. Eight weeks as well as 6 months after the baseline assessment, the participants of both groups will receive an email invitation to the postintervention or follow-up assessment.

Participants will not be financially compensated for participating in the study. However, participants in both 
study arms will receive free access to the intervention for 6 months after completing the follow-up assessments. In addition, participants who complete post-intervention and follow-up assessments will take part in a monthly drawing where they can win a universal $€ 50$ voucher that can be redeemed in a number of online stores.

Outcome data for participants who discontinue from intervention protocols will comprise the completed survey assessments prior to discontinuation as well as possible app usage data.

\section{Data management}

The study will be conducted in accordance with the EU General Data Protection Regulation (GDPR/DSGVO) and with the Berliner Datenschutzgesetz. The data will be collected using the MindDoc App and Unipark/EFS Survey. Both data collection tools comply with the GDPR and have implemented state-of-the art data protection measures. During data collection, data will be stored on the respective servers of these tools. On completion of the data collection, the data will be securely transferred to an encrypted data storage at Freie Universität Berlin. The data will exclusively be processed by authorised project staff. Fully anonymised data will be shared on request. Personal data will never be shared with third parties and will be deleted on completion of the trial to fully anonymise the data. Prior to the anonymisation, study participants can request the deletion of their data under their GDPR right. If a participant requests this, the data on Unipark will be deleted by the study team. Accounts and data on the MindDoc servers can be deleted in the settings of the app. Participant confidentiality will always be protected. All members of the research team will be required to maintain participant confidentiality and sign a confidentiality agreement. Only members of the Freie Universität Berlin will have access to the final trial dataset and will be responsible for the data analyses described below.

\section{Statistical methods}

Data will be characterised by descriptive statistical methods such as relative and absolute frequencies, mean, median, SD and IQR and appropriate graphics such as histograms, box plots and bar charts. Assumptions for the appropriate statistical tests will be checked for normality by histograms, skewness and Kolmogorov-Smirnoff test, sphericity will be assessed through Mauchly test epsilon corrections will be applied if sphericity cannot be assumed, and the assumption of equality of variancecovariance matrices will be investigated through Box test and Levene test.

Potential significant differences between the intervention and control group which may occur despite the randomisation will be detected by with $\chi 2$ and variance analyses on the baseline variables, appropriate statistical tests on the baseline variables. and accounted for in all analyses.
Participants will be excluded if they were missing data from their baseline assessments. The main outcomes will be examined with intention-to-treat analyses, with missing data imputed using baseline scores on symptom severity, mental health literacy, patient sovereignty, help seeking attitudes, quality of life, severity of personality dysfunction and demographic information. Given that there are four primary outcomes, we will impute using multivariate normal regression with an iterative Markov Chain Monte Carlo method based on initial treatment assignment. The pre-specified covariates and baseline measurement of primary endpoints will be added to the baseline model for improved precision. Potential bias due to non-random missing outcome observations will be addressed by estimating Random Forest Lee bounds. Concerns of multiple testing error will be addressed by bonferroni correction. To compare intervention effects on all primary and secondary outcomes, we will use analysis of covariance between groups at posttreatment and at follow-up adjusting for baseline scores. To address potential heterogeneity, intervention effects will exploratorily be estimated for subgroups (depression, anxiety, eating, somatoform and sleep-related disorders).

Sensitivity analyses will be performed exploiting different multiple imputation parameter settings, doing analyses with and without adjustment for baseline characteristics as well as by a per-protocol analysis excluding participants who violated the study protocol. The following protocol violations have been prespecified: (1) failure to download the app and complete the onboarding process, (2) use of the application before the randomisation date, (3) reporting of using of the MindDoc app during the intervention and follow-up period in the waitlist condition, (4) reporting of regular psychotherapy during the 8-week intervention period and (5) non-completion of postintervention or follow-up assessment.

\section{Ethics}

This trial will be conducted in compliance with the protocol, the Declaration of Helsinki and good clinical practice. The local ethical committee of FU Berlin has approved the protocol (AZ 039/2020). Amendments to the trial protocol will be immediately communicated to the local ethical committee as well as the trial registry by the corresponding author.

There will be no physical strain on the participants. The use of the MindDoc App requires a time expenditure of about 5 min per day for completing the assessment and additional time to engage with courses and exercises at the participants' convenience. These are not strains that exceed the usual level in studies with ambulatory assessment or mobile interventions. The app contains detailed information on how to access mental healthcare. Participants who report a high symptom load or functional impairment within the monitoring function of the app will be prompted to consult a healthcare professional within the automated feedback. Furthermore, individuals are repeatedly reminded that study participation does 
not substitute for diagnosis, counselling or treatment by a licensed physician or psychotherapist.

The questionnaire assessments could be perceived as a temporal emotional strain by some participants. The selection of questionnaires to assess primary and secondary outcomes was not only guided by psychometric properties, but also by the number of items in order to keep the burden at a minimum. In addition, individuals are informed that participation in all parts of the study is voluntary and can be terminated anytime without giving reasons.

Participants who report suicidal intent at baseline will be excluded from the study, but referred to crisis services (public telephone counselling) and provided with further information about treatment options. Participants who report suicidal intent at post-intervention or follow-up will be referred to crisis services (public telephone counselling, MindDoc counselling hotline) and provided with further information about treatment options. Participants who report suicidality at post or follow-up assessments will be recorded as an AE. If the 'crisis-bot' within the app is triggered, or if a participant scores $>1$ on the PHQ-9 suicidality item, this will be recorded as an AE. Other AEs and SAEs will be collected via self-report during the postintervention and follow-up assessments. Participants with severe symptoms will be excluded from participating, but receive detailed information on treatment options.

\section{Dissemination}

The MindDoc App will probably be available after the study on prescription by general practitioners for all German patients with mild to moderate internalising disorders within the framework of the Digitales Versorgungsgesetz ('digital healthcare act'), a new German law for digital health applications regulating the reimbursements of usage costs of digital health applications by German statutory health insurances.

The publication plan includes a main research report paper addressing the effects of the MindDoc App on all primary and secondary outcomes. In a second publication, predictors, moderators and mediators, such as personality functioning and negative life events, for the effectiveness of low-threshold mobile-based mental health interventions will be investigated. Results will be presented at national and international conferences. Authorship eligibility will be oriented on the respective contributions while no member of MindDoc will be involved in the data analysis. We also do not intend to use any professional writers.

\section{DISCUSSION}

Mental disorders pose a huge burden to both individuals and health systems. Many people who are affected suffer from more than one disorder. Thus, transdiagnostic approaches to screening, monitoring and treatment seem more suitable than disorder specific ones. Mental disorders often remain unrecognised and untreated.
This is largely caused by within-person barriers such as lack of trust in professionals or the desire to cope with problems without professional help. Although there are effective psychological treatments for all common mental health disorders, their availability is limited. Average waiting times for psychotherapy are almost 5 months.

The study will contribute both to the growing evidence base for closing gaps in mental healthcare through digital interventions and to the evidence base for low-threshold mobile-based interventions. With a focus on mental health literacy, patient sovereignty, and facilitation of access to care, this study will provide important insights into how mental healthcare seeking can be supported through low-threshold digital approaches. To date, no other study systematically investigated these direct mental health care-related effects of app-based interventions using an RCT design.

In addition, by including the assessment of potential moderators and predictors of treatment seeking behaviour and treatment response while at the same time assessing the treatments actually used, this study enables the investigation of possible indicators for decision making in stepped care.

Contributors AK and IB conceptualised and designed the study, wrote the first draft of the protocol and developed the statistical analysis plan. SB and CK and IB reviewed the manuscript, AK revised it based on these reviews and created the final version for submission.

Funding The Study is funded by MindDoc Health GmbH (Leopoldstraße 159, 80 804 Munich, Germany) within the framework of a scientific cooperation agreement between Freie Universität Berlin and MindDoc Health GmbH. AK, SB and CK have ultimate authority over study design; collection, management, analysis, and interpretation of data; writing of the report; and the decision to submit the report for publication.

Competing interests IB is the Chief Science Officer of MindDoc Health GmbH, the app manufacturer.

Patient consent for publication Not required.

Ethics approval The study was reviewed and has received ethics approval from the ethics committee of the Faculty for Psychology of the Freie Universität Berlin (AZ 039/2020) and has been registered within the German Clinical Trial register (www. drks.de, DRKS00022531).

Provenance and peer review Not commissioned; externally peer reviewed.

Supplemental material This content has been supplied by the author(s). It has not been vetted by BMJ Publishing Group Limited (BMJ) and may not have been peer-reviewed. Any opinions or recommendations discussed are solely those of the author(s) and are not endorsed by BMJ. BMJ disclaims all liability and responsibility arising from any reliance placed on the content. Where the content includes any translated material, BMJ does not warrant the accuracy and reliability of the translations (including but not limited to local regulations, clinical guidelines, terminology, drug names and drug dosages), and is not responsible for any error and/or omissions arising from translation and adaptation or otherwise.

Open access This is an open access article distributed in accordance with the Creative Commons Attribution Non Commercial (CC BY-NC 4.0) license, which permits others to distribute, remix, adapt, build upon this work non-commercially, and license their derivative works on different terms, provided the original work is properly cited, appropriate credit is given, any changes made indicated, and the use is non-commercial. See: http://creativecommons.org/licenses/by-nc/4.0/.

ORCID iD

André Kerber http://orcid.org/0000-0002-8588-7784 


\section{REFERENCES}

1 World Health Organization. Depression and other common mental disorders. Global health estimates, 2017. Available: https://apps. who.int/iris/bitstream/handle/10665/254610/WHO-MSD-MER-2017. 2-eng.pdf

2 Jacobi F, Höfler M, Strehle J. Psychische Störungen in Der Allgemeinbevölkerung: studie Zur Gesundheit Erwachsener in Deutschland und ihr Zusatzmodul Psychische Gesundheit (DEGS1MH). Nervenarzt 2014;85:77-87.

3 Schlack R, Hapke U, Maske U. Frequency and distribution of sleep problems and insomnia in the adult population in Germany: results of the German Health Interview and Examination Survey for Adults (DEGS1)]. Bundesgesundheitsblatt Gesundheitsforschung Gesundheitsschutz 2013;56:740-8.

4 Kessler RC, Bromet EJ. The epidemiology of depression across cultures. Annu Rev Public Health 2013;34:119-38.

5 Statista. Direkte Kosten psychischer Erkrankungen in Deutschland nACh Krankheitsart in den Jahren 2002 bis 2012 (in Milliarden Euro), 2018. Available: https://de.statista.com/statistik/daten/studie/ 246590/umfrage/direkte-kosten-psychischer-erkrankungen-indeutschland-nach-krankheitsart/

6 Kamp L, Pickshaus K. Regelungs/ücke psychische Belastungen schließen. Düsseldorf: Hans Böckler Stiftung, IG Metall; 2011.

7 Brown TA, Campbell LA, Lehman CL, et al. Current and lifetime comorbidity of the DSM-IV anxiety and mood disorders in a large clinical sample. J Abnorm Psychol 2001;110:585-99.

8 Haslam N, Holland E, Kuppens P. Categories versus dimensions in personality and psychopathology: a quantitative review of taxometric research. Psychol Med 2012;42:903-20.

9 Eaton NR, Krueger RF, Markon KE, et al. The structure and predictive validity of the internalizing disorders. J Abnorm Psychol 2013;122:86-92.

10 Krueger RF, Kotov R, Watson D, et al. Progress in achieving quantitative classification of psychopathology. World Psychiatry 2018;17:282-93.

11 Waszczuk MA, Eaton NR, Krueger RF, et al. Redefining phenotypes to advance psychiatric genetics: implications from hierarchical taxonomy of psychopathology. J Abnorm Psychol 2020;129:143-61.

12 Linardon J, Cuijpers P, Carlbring P, et al. The efficacy of appsupported smartphone interventions for mental health problems: a meta-analysis of randomized controlled trials. World Psychiatry 2019;18:325-36.

13 Blasko K, Ciulla R, Cavanagh R. Internet-Based Mental Health Interventions: Evidence, Practical Considerations, and Future Directions. In: Technology and mental health. Routledge, 2020: 38-55.

14 Kessler RC, Ormel J, Petukhova M, et al. Development of lifetime comorbidity in the world Health organization world mental health surveys. Arch Gen Psychiatry 2011;68:90.

15 Barlow DH, Sauer-Zavala S, Carl JR. The nature, diagnosis, and treatment of neuroticism: back to the future. Clinical Psychological Science 2014;2:344-65.

16 Weitz E, Kleiboer A, van Straten A, et al. The effects of psychotherapy for depression on anxiety symptoms: a metaanalysis. Psychol Med 2018;48:2140-52.

17 Barlow DH, Farchione TJ, Bullis JR, et al. The unified protocol for Transdiagnostic treatment of emotional disorders compared with Diagnosis-Specific protocols for anxiety disorders: a randomized clinical trial. JAMA Psychiatry 2017;74:875.

18 Murray LK, Dorsey S, Haroz E, et al. A common elements treatment approach for adult mental health problems in low- and middleincome countries. Cogn Behav Pract 2014;21:111-23.

19 Chisholm D, Knapp MRJ, Knudsen HC, et al. Client sociodemographic and service receipt inventory - European version: development of an instrument for international research. $\mathrm{Br} \mathrm{J}$ Psychiatry 2000;177:s28-33.

20 Clark DM, Canvin L, Green J, et al. Transparency about the outcomes of mental health services (IAPT approach): an analysis of public data. The Lancet 2018;391:679-86.

21 Mojtabai R, Olfson M, Sampson NA, et al. Barriers to mental health treatment: results from the National comorbidity survey replication. Psychol Med 2011;41:1751-61.

22 Andrade LH, Alonso J, Mneimneh Z, et al. Barriers to mental health treatment: results from the who world mental health surveys. Psychol Med 2014:44:1303-17.

23 Clement S, Schauman O, Graham T, et al. What is the impact of mental health-related stigma on help-seeking? A systematic review of quantitative and qualitative studies. Psychol Med 2015;45:11-27.

24 Gulliver A, Griffiths KM, Christensen H. Perceived barriers and facilitators to mental health help-seeking in young people: a systematic review. BMC Psychiatry 2010;10:113.
25 Schomerus G, Angermeyer MC. Stigma and its impact on helpseeking for mental disorders: what do we know? Epidemiol Psichiatr Soc 2008;17:31-7.

26 Halfin A. Depression: the benefits of early and appropriate treatment. Am J Manag Care 2007;13:S92-7.

27 Eichenberg C. Psychotherapie und Internet. Psychotherapeut 2011:56:468-74.

28 Musiat P, Tarrier N. Collateral outcomes in e-mental health: a systematic review of the evidence for added benefits of computerized cognitive behavior therapy interventions for mental health. Psychol Med 2014;44:3137-50.

29 Levin ME, Krafft J, Levin C. Does self-help increase rates of help seeking for student mental health problems by minimizing stigma as a barrier? J Am Coll Health 2018;66:302-9.

30 Simmons L, Jones T, Bradley E. Reducing mental health stigma: the relationship between knowledge and attitude change. EJMH 2017;12:25-40.

31 Farrer L, Christensen H, Griffiths KM, et al. Web-Based cognitive behavior therapy for depression with and without telephone tracking in a national helpline: secondary outcomes from a randomized controlled trial. J Med Internet Res 2012;14:e68.

32 Griffiths KM, Christensen H, Jorm AF, et al. Effect of webbased depression literacy and cognitive-behavioural therapy interventions on stigmatising attitudes to depression. Br J Psychiatry 2004:185:342-9.

33 Taylor-Rodgers E, Batterham PJ. Evaluation of an online psychoeducation intervention to promote mental health help seeking attitudes and intentions among young adults: randomised controlled trial. J Affect Disord 2014;168:65-71.

34 Christensen H, Leach LS, Barney L, et al. The effect of web based depression interventions on self reported help seeking: randomised controlled trial [ISRCTN77824516]. BMC Psychiatry 2006;6:13.

35 Klein B, Richards JC, Austin DW. Efficacy of Internet therapy for panic disorder. J Behav Ther Exp Psychiatry 2006;37:213-38.

36 Kravitz RL, Franks P, Feldman MD, et al. Patient engagement programs for recognition and initial treatment of depression in primary care: a randomized trial. JAMA 2013;310:310.

37 Johnson JA, Devdutt J, Mehrotra S, et al. Barriers to professional help-seeking for distress and potential utility of a mental health APP components: Stakeholder perspectives. Cureus.

38 Burchert S, Kerber A, Zimmermann J, et al. Screening accuracy of a 14-day smartphone ambulatory assessment of depression symptoms and mood dynamics in a general population sample: comparison with the PHQ-9 depression screening. PLoS One 2021;16:e0244955

39 Kotov R, Krueger RF, Watson D. A paradigm shift in psychiatric classification: the hierarchical taxonomy of psychopathology (HiTOP). World Psychiatry 2018;17:24-5.

40 Kroenke K, Spitzer RL, Williams JB. The PHQ-9: validity of a brief depression severity measure. J Gen Intern Med 2001;16:606-13.

41 Kroenke K, Spitzer RL, Williams JBW, et al. Anxiety disorders in primary care: prevalence, impairment, comorbidity, and detection. Ann Intern Med 2007;146:317.

42 Kroenke K, Spitzer RL, Williams JBW. The PHQ-15: validity of a new measure for evaluating the severity of somatic symptoms. Psychosom Med 2002;64:258-66.

43 Crönlein T, Langguth B, Popp R, et al. Regensburg insomnia scale (RIS): a new short rating scale for the assessment of psychological symptoms and sleep in insomnia; study design: development and validation of a new short self-rating scale in a sample of 218 patients suffering from insomnia and 94 healthy controls. Health Qual Life Outcomes 2013;11:65.

44 Zimmermann J, Kerber A, Rek K, et al. A brief but comprehensive review of research on the alternative DSM-5 model for personality disorders. Curr Psychiatry Rep 2019;21:92.

45 Bach B, Kerber A, Aluja A, et al. International assessment of DSM-5 and ICD-11 personality disorder traits: toward a common nosology in DSM-5.1. Psychopathology 2020;53:179-88.

46 Ehrenthal JC, Dinger U, Schauenburg H, et al. Entwicklung einer Zwölf-Item-Version des OPD-Strukturfragebogens (OPDSFK)/ Development of a 12-item version of the OPD-Structure Questionnaire (OPD-SQS). Zeitschrift für Psychosomatische Medizin und Psychotherapie 2015;61:262-74.

47 Kerber A, Schultze M, Müller S, et al. Development of a short and ICD-11 compatible measure for DSM-5 maladaptive personality traits using ant colony optimization algorithms. Assessment 2020:1073191120971848.

48 Richardson J, lezzi A, Khan MA, et al. Validity and reliability of the Assessment of Quality of Life (AQoL)-8D multi-attribute utility instrument. Patient 2014;7:85-96. 
49 Dias $\mathrm{P}$, Campos L, Almeida $\mathrm{H}$, et al. Mental health literacy in young adults: adaptation and psychometric properties of the mental health literacy questionnaire. Int J Environ Res Public Health 2018;15:1318.

50 Houle J, Gascon-Depatie M, Bélanger-Dumontier G, et al. Depression self-management support: a systematic review. Patient Educ Couns 2013;91:271-9.

51 Morgan AJ, Jorm AF. Self-Help strategies that are helpful for subthreshold depression: a Delphi consensus study. J Affect Disord 2009;115:196-200.

52 van Grieken RA, Kirkenier ACE, Koeter MWJ, et al. Patients perspective on self-management in the recovery from depression. Health Expect 2015;18:1339-48.

53 Villaggi B, Provencher $\mathrm{H}$, Coulombe S, et al. Self-Management strategies in recovery from mood and anxiety disorders. Glob Qual Nurs Res 2015;2:233339361560609.

54 Saltman RB. Patient choice and patient Empowerment in northern European health systems: a conceptual framework. Int J Health Serv 1994;24:201-29.
55 Mackenzie CS, Knox VJ, Gekoski WL, et al. An adaptation and extension of the attitudes toward seeking professional psychological help Scale1. J Appl Soc Psychol 2004;34:2410-33.

56 Boß L, Lehr D, Reis D, et al. Reliability and validity of assessing user satisfaction with web-based health interventions. J Med Internet Res 2016;18:e234.

57 Holmes $\mathrm{TH}$, Rahe $\mathrm{RH}$. The social readjustment rating scale. $J$ Psychosom Res 1967;11:213-8.

58 Hobson CJ, Kamen J, Szostek J, et al. Stressful life events: a revision and update of the social readjustment rating scale. Int J Stress Manag 1998;5:1-23.

59 Linardon J, Fuller-Tyszkiewicz M. Attrition and adherence in smartphone-delivered interventions for mental health problems: a systematic and meta-analytic review. J Consult Clin Psychol 2020;88:1-13.

60 Schalet BD, Cook KF, Choi SW, et al. Establishing a common metric for self-reported anxiety: linking the MASQ, PANAS, and GAD-7 to PROMIS anxiety. J Anxiety Disord 2014;28:88-96. 